\title{
Doing More with Less: A Nice and Useful Idea!
}

W e have just read with attention the interesting and useful article by Drs. Peacock and Timpone ${ }^{1}$ describing the accuracy of spinal CT in suspected cauda equina syndrome.

The authors described the use of lumbar spinal CT to demonstrate cauda equina compression in patients with severe low back pain, motor or sensory deficit, and reduced rectal sphincter tone.

We agree with the authors that in most cases, only university hospitals have the possibility to ensure a 24/7 MR imaging service.

It is also true that in most hospitals, a CT is not performed as the first-line imaging, MR is directly done, and, in some cases, CT is performed after the MR imaging, though it is CT that will finally provide the key to the diagnosis (Fig 1).

On the other hand, we have noticed over the past few years that our younger residents and clinicians have become slightly less proficient in reading a spinal CT and overrate the superiority of MR imaging in relation to CT when, in reality, both techniques are complementary. In addition, we are facing the fact that in patients with pain, the quality of MR imaging is often mediocre because of kinetic artifacts. MR imaging in some of these patients may also be problematic because the whole organizational process around it takes a lot of time, and, in a few cases, even an intervention by anesthesiologists is necessary; this all could be avoided by performing a quick CT scan of the spine, which could help to either provide the diagnosis or at least exclude a clinically relevant pathology. Also, the presence of blood in the spinal canal, or around it in the case of trauma, can produce susceptibility artifacts, ${ }^{2}$ which are going to make an easy diagnosis difficult, especially for a less experienced resident. We can only note that these inappropriately prescribed MRIs have increased markedly over the past few years, which has led to an increase in the cost of the management of these patients for whom an MRI might not be necessary.

Finally, we agree with the authors that MR imaging has a superior soft-tissue contrast resolution, but we also believe that, despite this, CT can provide the diagnosis in some cases.

\section{REFERENCES}

1. Peacock JG, Timpone VM. Doing more with less: diagnostic accuracy of CT in suspected cauda equina syndrome. AJNR Am J Neuroradiol 2016 Oct 27. [Epub ahead of print] CrossRef Medline

2. Vargas MI, Delavelle J, Kohler R, et al. Brain and spine MRI artifacts at 3Tesla. J Neuroradiol 2009;36:74-81 CrossRef Medline

(D) M.I Vargas

Division of Neuroradiology

(1) A. Platon

Division of Radiology

(D). Boto

(D) K.-O. Lovblad

Division of Neuroradiology

Department of Radiology and Medical Informatics Geneva University Hospital Geneva, Switzerland 


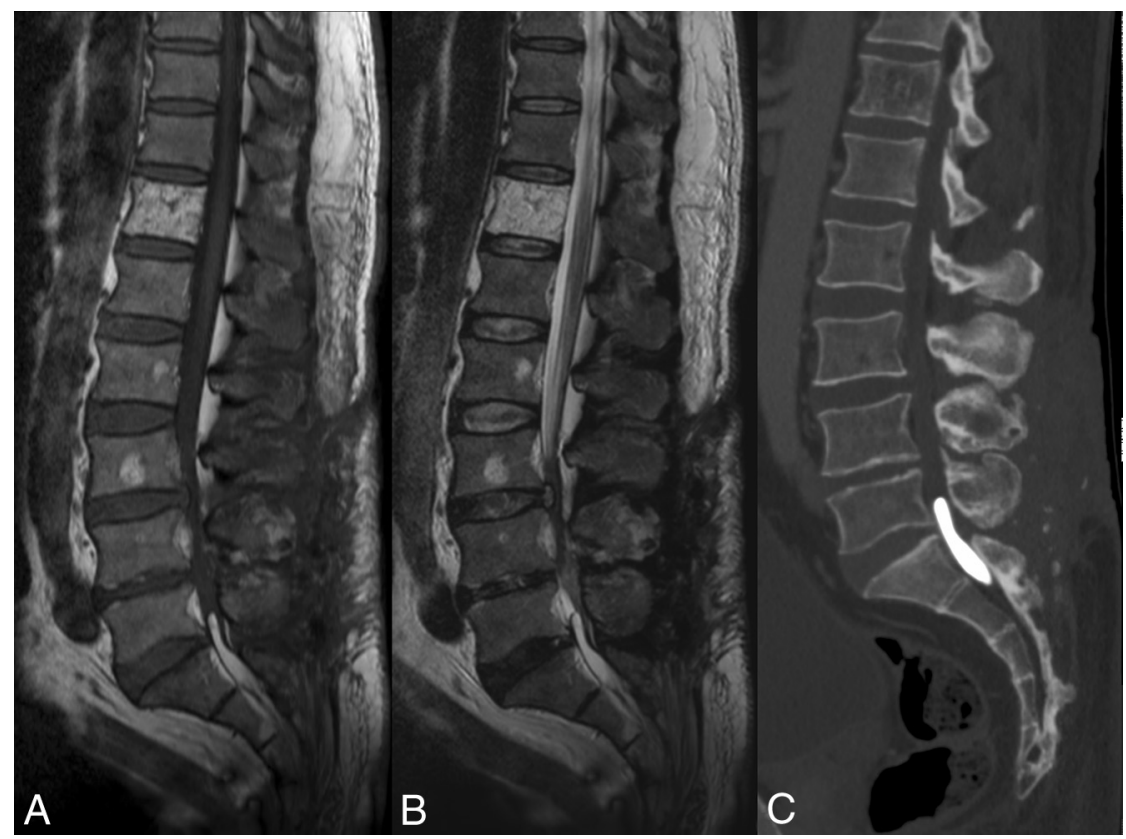

FIG 1. Sagittal spin-echo TIWI, FSE T2WI, and MPR of CT. Note the hyperintensity on T1 in the spinal canal at level of S1; very difficult to detect on $\mathrm{T} 2$ and easily identified on the $\mathrm{CT}$, corresponding to lipiodol in a patient with suspected cauda equina syndrome. 